\title{
Study on Composition and Development of the Database Management
}

\section{System}

\author{
Fenfen $\mathrm{Wu}^{1}$ \\ ${ }^{1}$ Henan Vocational and Technical College of Communications, Henan, 450000 \\ 346591653@163.com
}

\section{KEYWORDS: Database; Management System; System Compostion}

\begin{abstract}
The database is long-term storage in the computer, organized, shared data collection. Database to store large amounts of information in an effective and quick access to play an important role, it is the core and foundation of large information systems. Database management system is to establish, use and maintenance of the database management established. This paper briefly describes the four major components of the database management system, we analyze the trends and directions of database technology.
\end{abstract}

\section{Introduction}

The database is long-term storage in the computer, organized, shared data collection. Database to store large amounts of information in an effective and quick access to play an important role, it is the core and foundation of large information systems. Database management system is to establish, use and maintenance of the database management established. This paper briefly describes the four major components of the database management system, we analyze the trends and directions of database technology. Database management system can achieve operation of the database functions, including the following four aspects: to retrieve, insert, delete, and modify. Database management system to achieve the operating performance of the database must contain the compiler that DML. DML includes two types, one for the plug-in, and the other is stand-alone.

Database management system controls the database is mainly reflected in the following aspects:

Security control function of the database management system is to protect the database. When the data in the database is not authorized, this feature prevents the data in the database is modified to prevent the integrity of the database is destroyed, thus preventing the loss caused to the user database. Integrity control function database management system on the other hand protect the database to ensure accurate and consistent data. Integrity control database management system, the data in the database can not be modified protection, so as to maintain the integrity of the data in the database, the database management system control function by the mandatory implementation. This control is an advantage of database technology, data can be shared, it will not cause damage to the data, thus ensuring the accuracy of the data in the database. Concurrency Control database management system can achieve multi-tasking, multi-user operating situation, in the above case also ensures the integrity and accuracy of the data. In the operation of the database will occur all sorts of unexpected condition occurs, to ensure the normal operation of the database, the database management system with the need to restore control. This control function can create multiple backup database applications, can restore the role of the original data. 


\section{Composition of Database Management System}

Database system refers to the organization, and maintenance of human-machine access large amounts of data management systems, is an organic whole by the computer, database, database management systems and related personnel, which is organized in the form of data organization, storage, management and other functions, database and database management system for the organization, management and processing of information provided premise. Database management system normal operation of the database to improve the guarantee. Database users tend to only deal with the database through the database management system software tools. In database management systems, application can not get the required data directly from the storage medium, it must first submit a request to the database management system, the database management system is responsible for retrieving data from a storage medium and provided to the application. Therefore, a database management system is the interface between the application and data. Database management system is a data management software system, the core of the database system. Database management system to achieve the creation, use and management of the database, its features include: easy and accurate input of raw data. Reliability and accuracy of management system from the correct original data entry. In the definition of the logical structure of the database information, while also improving the overall constraint, the constraint is to ensure the overall quality of a rule. A series of text in the database project variety, type, and other test work, you can click the drop-down box to enter simplifies operation of testing personnel, to avoid making mistakes; the system input data integrity, accuracy efficacy, in the event of an error the system will automatically alarm. Practical and convenient data editing functions. Database management system data processing subsystem to help users to add, modify, delete database information, and help users tap valuable information in the database. Flexible query search function. According to field values, query the database to meet the conditions of the recording, you can check product quality information at any period of time; you can also browse sequence information. System management functions. DBMS data management subsystem management database functionality.

Database management system typically consists of the following four parts.

Data Definition Language and Translation handler database management systems typically have to provide the data definition language (data definitionlanguage, referred DDL) for various modes of user-defined database, the translator is responsible for translating them into the corresponding internal representation, which generated target mode.

Data manipulation language and compiler (or interpreted) program database management system provides data manipulation language (data manipulation language, called DML) realize a database retrieval, insert, modify, delete and other basic operations. DML into the host type DML and autonomous DML categories.

Database operation control program database management system provides system operation control program is responsible for running the database control and management, which monitors all operations on the data in the process database operation, the control management database resources to deal with the multi-user concurrent operation . Database management systems also typically provide some utility, database users can use these utilities to complete the establishment and maintenance of the database, as well as conversion and communication data format.

\section{The Application of Database System}

Information is the modern social enterprise an irreversible trend, the database management system is the core of enterprise information. In the process of information, computer and other 
information technology has become an indispensable foundation system platform, platform information and link up conventional operations, the most important intermediary is the database management system. Common patterns in the enterprise database in the actual business applications, the most common are two: one is the information processing resources, and the other is the information network services. Database management system in the enterprise database management system common technique commonly used technology in the enterprise customers with mission architecture, distributed databases, Web database. Web database is an excellent example of a database technology and network technology, but also a network database, usually presents three database schema: database, middle Web service, front-end browsing. Enterprise information applications, database management systems and application development provides a great space, a lot of new database technologies are developed from practical application in conjunction with, and now enterprise information application in the content more widely, far beyond the early production data access management process, but the information will be extended to apply to all activities and outcomes, such as enterprise document graphical database management. Database Support Decision of As information technology widely used in various fields, people in the information process has been established to millions of information systems. Since the human ability to continuously enhance access to data, data, and also a rapid increase in the size of the database, these data are a valuable resource, people in the use of database management systems work, have realized the hidden data with valuable knowledge and law, and focus on database technology decision support research and development. Enterprise data management information system has been established in the business reflects the current operating status, required by the various functional departments, the use of these data can be effectively accomplish everyday tasks contained in enterprise management, the general application of such a database called online transaction processing (OLTP). In the decision-making required data online analytical processing (OLAP), which is to focus on meeting the needs of decision-makers and senior management, the flexibility to quickly perform complex queries and analyzes large amounts of data, and in a clear and intuitive the form of the result to the decision-makers, so that they accurately grasp the business situation, understand the market dynamics, to develop the right programs to increase enterprise efficiency.

Centralized database management system development trend database management system development. With the development of science and technology, database technology is also showing a wide variety of types, database products are also increasingly diversified. With the continuous development of database products, and the growing tendency of centralized database management systems, database technology is gradually centralizing development, centralized database management system and centralized database technology-related new products continue to come to promote.

Database management system typically consists of the following four parts.

Data Definition Language and Translation handler database management systems typically have to provide the data definition language (data definitionlanguage, referred DDL) for various modes of user-defined database, the translator is responsible for translating them into the corresponding internal representation, which generated target mode.

Data manipulation language and compiler (or interpreted) program database management system provides data manipulation language (data manipulation language, called DML) realize a database retrieval, insert, modify, delete and other basic operations. DML into the host type DML and autonomous DML categories.

Database operation control program database management system provides system operation control program is responsible for running the database control and management, which monitors 
all operations on the data in the process database operation, the control management database resources to deal with the multi-user concurrent operation . Database management systems also typically provide some utility, database users can use these utilities to complete the establishment and maintenance of the database, as well as conversion and communication data format.

\section{Conclusion}

Database management system development of high-end. Development of a database management system is constantly promote the development of society as a whole, in an increasingly competitive situation, to promote information technology continues to advance, the need to constantly improve the database management system to improve database management position in society. For database management system becoming more and more high-end development, database technology can make to the world stage, resulting in a more advanced information technology to enhance the level of information systems. To be able to do a lot more processing and control of information technology to further enhance the database. Information service levels need to improve, and even business management and decision-making, also need to constantly improve.

Theory and technology of the database. The database theory and technology foundation is to support the development of database management systems. Database theory and technology are mature state, whether it is technical or theoretical model database technology products, they are increasingly vigorous development and maturation. Database theory and technology also need to be constantly updated, its replacement will bring growing database products. There will be development of different information technologies in different applications, in addition to the information technology, other information technology is constantly evolving, such as middleware. Development of other information technology will broaden the application of database technology, database technology makes more flexible in use more centralized.

The development of object databases, distributed database, knowledge base database technology will develop more and more dependent on their maturity and common database basic theory of technology. For example, new data model theory of the formation of mature technology products, as a mature relational database theory knowledge to promote the development of a relational database, the new database will also promote the great development of the basic theory of database technology. The database will continue to improve in the high reliability, high performance, high scalability and high security, combined with the new technology data between different databases in terms of interoperability of Internet connectivity, network database development using Network Data Representation technology. There are different techniques in different application data will be used to support collaborative applications existing database technology, such as middleware, so you can expand the application of database technology, the formation of flexible and integrated database technology.

\section{REFERENCE:}

[1] Jimei Xing. Fuze design and simulation data management systems and databases Study [D] .Tsinghua University, 1999.

[2] Xu Ping. grid design and implementation of database management system query optimization [D]. Zhejiang University, 2005.

[3] Yuanpei Sen. Memory Database Design and Implementation [D]. Nanjing University of Aeronautics and Astronautics, 2006. 
[4] Li Jingjing. Distributed Database Management Information System [D]. Wuhan University of Technology, 2004. 